\title{
Evolutionary Synthesis Modelling of Young Star Clusters in Merging Galaxies
}

\author{
Peter Anders, Uta Fritze - v. Alvensleben \\ Universitäts-Sternwarte Göttingen, Geismarlandstr. 11, 37083 Göttingen, \\ Germany \\ Richard de Grijs \\ Institute of Astronomy, Madingley Road, Cambridge CBЗ OHA, UK
}

\begin{abstract}
The observational properties of globular cluster systems (GCSs) are vital tools to investigate the violent star formation histories of their host galaxies. This violence is thought to have been triggered by galaxy interactions or mergers. The most basic properties of a GCS are its luminosity function (number of clusters per luminosity bin) and color distributions.

A large number of observed GCS show bimodal color distributions, which can be translated into a bimodality in either metallicity and/or age. An additional uncertainty comes into play when one considers extinction.

These effects can be disentangled either by obtaining spectroscopic data for the clusters or by imaging observations in at least four passbands. This allows us then to discriminate between various formation scenarios of GCSs, e.g. the merger scenario by Ashman \& Zepf, and the multi-phase collapse model by Forbes et. al.

Young and metal-rich star cluster populations are seen to form in interacting and merging galaxies. We analyse multiwavelength broad-band observations of these young cluster systems provided by the ASTROVIRTEL project.
\end{abstract}

Keywords: Globular clusters: general, open clusters and associations: general, galaxies: star clusters, galaxies: evolution

\section{Modelling multi-colour star cluster data}

We have further extended the Göttingen evolutionary synthesis (ES) code by including the effects of gaseous emission. The gaseous emission contributes significantly to the integrated light of stellar populations younger than $10^{8}$ years (Anders et. al., 2002a, in prep.); see Figure 1. In addition, the effect of internal dust extinction has been included.

The simultaneous determination of a cluster's age, metallicity, extinction and mass is achieved by comparing an appropriate grid of ES models with the observed spectral energy distributions (SEDs) in a least-squares sense. Due to the well-known age-metallicity degeneracy (and a similar age-extinction degeneracy) at optical wavelengths (and the dependence on the mass of the cluster), the use of multi-passband observations is essential to determine these parameters independently.

(C) 2018 Kluwer Academic Publishers. Printed in the Netherlands. 

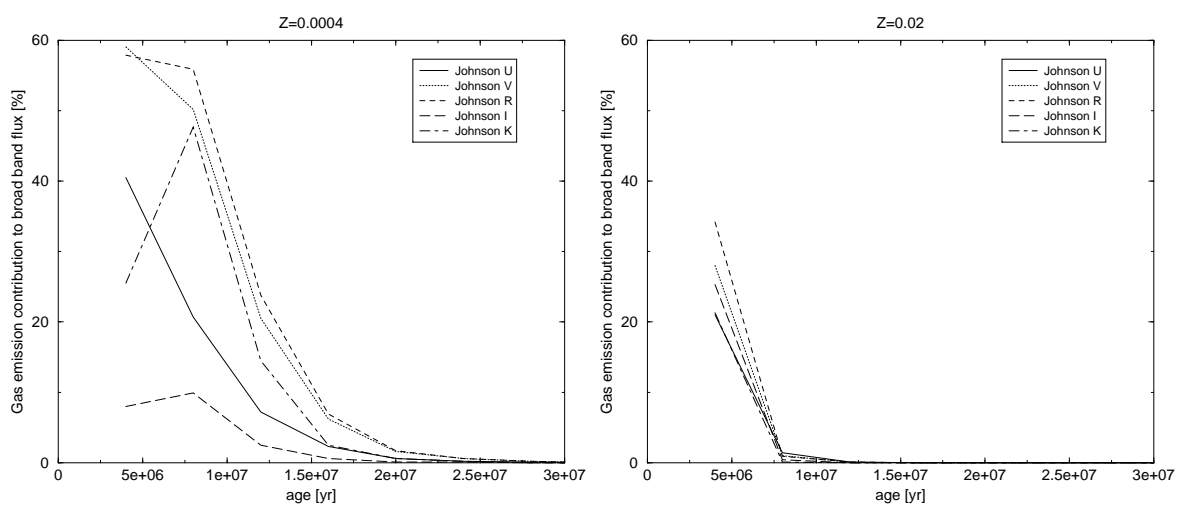

Figure 1. Time evolution of the gaseous emission contribution to broad-band fluxes in Johnson passbands $U, B, V, I$, and $K$ at low metallicity $\mathrm{Z}=0.0004$ (left panel) and solar metallicity (right panel).

\subsection{Results for Artificial Clusters}

We constructed five artificial clusters with known properties, i.e., solar metallicity, extinction $E(B-V)=0.1$, ages of 8,60, and $200 \mathrm{Myr}$, and 1 and 10 Gyr. The SEDs for these artificial clusters were taken from our model grid, and a typical observational error of 0.1 mag was assumed. Subsequently, we redetermined the best-fit cluster parameters using our ES code, and compared them to the original values. We constructed a number of additional clusters characterized by color offsets (too red or too blue by $0.1 \mathrm{mag}$ ) to assess the accuracy of our model fits. From this study we conclude that

1. The choice of passband combination required for a proper determination of the basic cluster parameters (age, metallicity, extinction) can be optimized towards the expected mean parameter values.

2. In order to determine reliable ages, the use of ultraviolet (UV) passbands is vital for all ages. Including a near-infrared (NIR) passband improves the accuracy further.

3. For metallicity determinations, NIR observations are most important, while the UV is essential for young systems (ages $\leq 100$ Myr).

4. The extinction is best determined using UV + optical colors.

In general, the accuracy and the best passband combination are a strong function of the age of the cluster's stellar population (Anders et. al., 2002b, in prep.). The availability of observations spanning the entire 
wavelength range, from UV to NIR, allows to constrain all parameters (age, metallicity and internal extinction) most efficiently.

\section{Application and first results: NGC 1569}

The irregular dwarf galaxy NGC 1569 is commonly classified as a (post) starburst galaxy with HII region metallicity $\sim(0.2-0.5) Z_{\odot}$. Highresolution multipassband imaging data were provided by the ESO / ST-ECF ASTROVIRTEL project "The Evolution and Environmental Dependence of Star Cluster Luminosity Functions" (PI R. de Grijs). In addition to the two well-studied super star clusters in its main disk, they reveal a number of fainter objects resembling compact star clusters (see also (Hunter et. al., 2000)).

Our fitting algorithm provides evidence for continuous star formation starting at least 7 Gyr ago. The recent burst, however, started around 80 Myr ago, with a possible peak of cluster formation around 35 Myr ago and a major peak in the cluster formation rate in the youngest age bin $\leq 8 \mathrm{Myr}$ (see Figure 2). These results are consistent with previous results (Greggio et. al., 1998; Aloisi et. al., 2001) regarding the bulk of the starburst activity in general and the formation of the two super star clusters in particular. As seen in previous studies, the most recent, enhanced epoch of star formation coincides well with the formation of morphologically peculiar features, like $\mathrm{H} \alpha$ filaments and "superbubbles" (Waller, 1991).
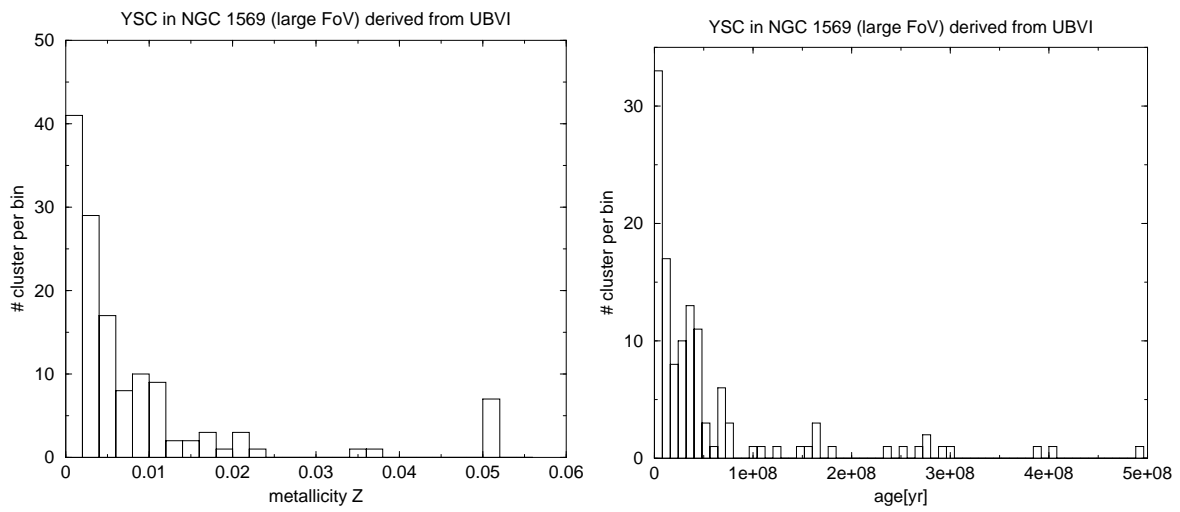

Figure 2. Left panel: Metallicity distribution of young star clusters in NGC 1569, derived from $U, B, V$ and $I$; right panel: corresponding age distribution (during the burst; nine older clusters, up to 7 Gyr old, are also present).

An accompanying Hi cloud is located at $5 \mathrm{kpc}$ from NGC 1569, connected to it by an $\mathrm{HI}$ bridge, suggesting a tidal interaction as a possible cause for the starburst (Stil \& Israel, 1998). 
We determine masses for the individual star clusters on the basis of our model mass-to-light ratios at their respective ages and metallicities. We find masses up to $10^{6} M_{\odot}$. The metallicity distribution is broader than found in previous surveys, which mainly focussed on the ISM (Kobulnicky \& Skillman, 1997; Devost et. al., 1997). This may imply different chemical enrichment processes in the star clusters vs. the ISM, or selection effects caused by the different spatial coverage. The internal dust extinction is found to be low $(E(B-V)<0.25$, with the majority of the clusters being affected by $E(B-V)<0.1$ ) (Anders et. al., 2002c, in prep.).

\section{Acknowledgements}

Very efficient support given by ASTROVIRTEL, a Project funded by the European Commission under FP5 Contract No. HPRI-CT-199900081, is gratefully acknowledged. P. Anders is partially supported by DFG grant DFG Fr 916/11.

\section{References}

Anders, P., Fritze-v.Alvensleben, U., 2002a, in prep.

Anders, P., Fritze-v.Alvensleben, U., de Grijs, R., 2002b, in prep.

Anders, P., de Grijs, R., Fritze-v.Alvensleben, U., 2002c, in prep.

Aloisi, A., Clampin, M., Diolaiti, E. et. al., 2001, AJ 121, 1425

Devost, D., Roy, J.-R., Drissen, L., 1997, ApJ 482, 765

Greggio, L., Tosi, M., Clampin, M., de Marchi, G., Leitherer, C., Nota, A., Sirianni, M., 1998, ApJ 504, 725

Hunter, D. A., O'Connell, R. W., Gallagher, J. S., Smecker-Hane, T. A., 2000, AJ 120,2383

Kobulnicky, H. A., Skillman, E. D., 1997, ApJ 489, 636

Stil, J. M., Israel, F. P., 1998, A\&A 337, 64

Waller, W. H., 1991, ApJ 370, 144 